MUZIKOLOŠKI ZBORNIK - MUSICOLOGICAL ANNUAL XIV, LJUBLJANA 1978

UDK 786.2(430) Schumann

\title{
NEUE SCHUMANNIANA
}

Wolfgang B o et ticher (Göttingen)

"Unseren Kindern zu treuer Aufbewahrung, Dresden, den 13ten Juni 1845" schrieb Robert Schumann auf dem ersten Blatt eines "Familien-Albưms", darunter zeichnete "Clara Schumann" mit eigener Hand. Diese kostabare Sammlung eines Künstlerehepaares wurde vom Verfasser zwar 1939 kursorisch im Rahmen eines quellenkritischen Anhangs erwähnt, ${ }^{1}$ ein jüngerer Bericht ist gefolgt. ${ }^{2}$ Gleichwohl war es seither nur möglich, wenige Texte bekannt zu machen. ${ }^{3}$ Der Enkel des Komponisten, Ferdinand, bot 1934 der Sächsischen Landesbibliothek Dresden das "Album" zum Kauf an, es wurde am 19. 2. 1935 erworben. ${ }^{4}$ Die heute unter der Signatur $373^{a}$ in einzelnen Mappen sorgsam aufbewahrte Sammlung bestand, wie sich der Verfasser bei seinem Studium 1936-1938 aufgezeichnet hat, aus einer Kassette im Querformat $26 \times 37 \mathrm{~cm}$, die ca. $9 \mathrm{~cm}$ Höhe maß. Das Äußere war mit schwarzem Samt beklebt, oben auf dem Deckel waren die Namen Robert und Clara in Gold eingepreßt. Im Innern, mit weißem Moiré-Textil ausgelegt, befanden sich 110 braune Kartons (mit Goldschnitt), auf denen die Dokumente aufgeklebt waren. Man unterschied offenbar einen repräsentativen Teil (mit Autographen von J. S. Bach, W. A. Mozart etc.) von Papieren aus dem engeren Familienbereich, die z. T. in kleinen Kouverts verborgen waren, mit persönlichen Aufschriften. Mehrere Objekte waren mit rotseidenen Bändern zusammengebunden oder durch Schleifen verschlossen (Blumen, Blätter, Haarlocken, sonstige Reliquien). In den Brandnächten Februar 1945 befand sich die Sammiung

${ }^{1}$ Verfasser, R. Schumann, Einführung in Persönlichkeit und Werk..., Berlin 1941 (Phil. Diss. Berlin 1939), S. 628.

${ }^{2}$ Verfasser, in: Scritti in onore di Luigi Ronga, Rom-Milano 1973, S. $36 \mathrm{ff}$.

${ }^{3}$ Verfasser, R. Schumann in seinen Schriften und Briefen, Berlin 1942, dort namentlich die Bildtafeln VIII ff.

${ }^{4} \mathrm{M}$. Bollert in: Jahresbericht der Sächsischen Landesbibliothek Dresden, Dresden 1936 , S. 41. 
im Tiefenkeller des gänzlich zerstörten "Japanischen Palais» (der ehemaligen Musiksammlung der Landesbibliothek), entging zwar der Vernichtung, wurde aber durch das eingedrungene Elbwasser schwer beeinträchträchtigt: der Einband ging zugrunde, die Tintenschrift verwischte, das Wasser zersetzte das dünne Papier, verklebte die Lagen, einiges fiel der Dispersion ganz zum Opfer. Nach früh einsetzender Restaurierung erstattete in dankenswerter Weise $\mathrm{Ch}$. Boden ${ }^{5}$ einen Bericht, dem ein vorläufiges Verzeichns der verbliebenen Objekte angeschlossen ist. Der Verfasser hat aufgrund seiner genannten alten Aufzeichnungen seit 1959 die Reste geordnet und wenige alte Fotografien zur Entzifferung der Brieftexte mit einsetzen können. Der gesamte Briefbestand wird durch ihn in Kürze im VEB Deutscher Verlag für Musik in Leipzig (DDR) veröffentlicht, verbunden mit einem wissenschaftlichen Kommentar, der das verstreute biographische Material eines auch als Schriftsteller hervorragend wirksamen Komponisten möglichst lückenlos $\mathrm{zu}$ rekonstruieren sucht.

So schwierig sich die Sichtung der Dokumente durchführen ließ, Schumanns autographes "Verzeichniß der Handschriften" (4 Blätter) bezeugt, wie sorgsam der Meister die Sammlung aufbaute. Nach der Schrift zu schließen dürfte der Index 1847-1848 angelegt worden sein, als terminus ergeben sich Nachträge mit spitzerer Feder: "Bendemann, E." (der Brief dieses befreundeten Malers ist 20. 9. 1847 datiert) und "Eichendorff, J. v." (das Gedicht ist 20. 2. 1847 gezeichnet). Der Index weist mehrere Namen auf, von denen die Briefstücke nicht mehr überliefert sind. Einige Einsender sind bisher in ihren Beziehungen zu Schumann gänzlich unbekannt, da ihr Name in der sogenannten "Correspondenz" (siehe unten) nicht vertreten ist. Schumann hat einige Namen unterstrichen: Beethoven, Chopin, Liszt, Mendelssohn, Lind, Mozart, Paganini, Goethe, Berlioz, Eichendorff, Haydn, Gade, Herder, Jean Paul, Rossini, Rückert, Schubert, Schroeder-Devrient, Spontini, Thorwaldsen, Weber. Richard Wagner ist nicht unterstrichen. Einige Kontrolle gewährt ein "Inhaltsverzeichnis der von Robert und Clara Schumann gesammelten Briefe», das als Register beim Verkauf 1936 von dem Enkel vorlag; dieser Nachkomme, Ferdinand Schumann, hat in einem weiteren Verzeichnis wertvolle Hinweise $\mathrm{zu}$ den einzelnen Briefeinsendern hinterlassen, auch eine Liste der "fehlenden" Stücke Beigefügt, von denen aber einige der Verfasser im Konvolut wieder hat anfinden können. Unmittelbar vor dem Verkauf gelangten einige Autographe in anderen Besitz, unter diesem Bestand befanden sich Stuicke von J. S. Bach, Briefe Mozarts an seine Gattin, Autographe von Beethoven, Haydn, Wagner, Chopin, Herder, Rossini, Schubert, Salieri. Die Gesamtliste zeigt 52 übersprungene Nummern, mithin etwa ein Viertel des Bestands, wie ihn Schumann hinterlassen hatte.

${ }^{5}$ Ch. Boden, Das Schumann-Album, eine Kostbarkeit der Sächsischen Landesbibliothek in Dresden, s. e. et 1. (Dresden 1956). Beschreibung S. 1-3. Zuvor hatten gemäß Bearbeitungsblatt der Bibliothek lediglich Einsicht genommen: 22. 6 . 1937 Felix Schumann (Urenkel) und ab 19. 8. 1937 der Verfasser. 
Im Gegensatz zu J. Brahms hat Schumann jeden Fetzen Papier als Schaffensciokument aufbewahrt und mit Akribie gesammelt. Er dürfte schon mehrere Jahre vor der Eheschließung sein geheimes Brevier aufgebaut haben, wahrscheinlich waren es zuerst Briefe seiner Lehrer, die ersten anerkennenden Stimmen, familiäre Schrifzeugnisse. 1834, mit Beginn der Redaktionsarbeit und seiner "Correspondenz", war der Grundstock der Sammlung gelegt. In der Ehejahren weitete sich der Blick auf die Nachkommen und manches Objekt wurde aus repräsentativen Gründen, weniger aufgrund interner Erwägungen aufgenommen. Der persönlichste Bereich, der Briefwechsel mit Clara, seiner Braut, wurde gänzlich an anderem Ort aufbewahrt. Nach 1842 stieg die Zahl seiner Weggenossen, mit denen er in geistigem Austausch stand, gewaltig. Insofern ergeben sich für den Schriftsteller und Komponisten Schumann beim Studium dieser Papiere z.T. ganz neue Einsichten. Von großem Wert sind auch Erinnerungsblätter, Dedikationspapiere. Mehrere Zeichnungen aus dem Freundeskreis sind noch unbekannt. Mit der Eheschließung 1840 hat die Gattin ihre eigene Sammlung, meist musikalische Albumblätter, mit Schumanns Bestand vereinigt. Dabei wird deutlich, daß der ältere Teil der Papiere Claras nicht selten dem stilistischen Milieu Schumanns widerspricht, da die junge Virtuosin sich in einem Personenkreis befand, der von Friedrich Wieck gefördert wurde. Im übrigen hat Clara nach dem Tode ihres Gatten weitergesammelt und den unterschiedlichen Charakter des Konvoluts noch vertieft.

Zur Identifikation der Briefeinsender und zur näheren Bestimmung der Briefinhalte hat ter Verfasser zwei wichtige Quellen konsultieren können: die "Correspondenz" und das "Briefbuch". Bei der "Correspondenz" handelt es sich um sämtliche Briefe, die Schumann seit 1834, dem Gründungsjahr der "Neuen Zeitschrift für Musik», empfangen hat. Fast 5000 Briefe in laufender Numerierung wurden in 28 Bänden vereinigt. Von diesem Bestand, der seit 1945 verschollen ist (ehemals in der Staatsbibliothek Berlin), hat der Verfasser zahlreiche Kopien zurückbehalten und zum Vergleich einsetzen können. Ergänzend war das "Briefbuch" zu konsultieren: Schumanns Verzeichnis sämtlicher von ihm abgesandter Briefe, mit sehr wertvollen kurzen Notizen über den Inhalt der Schreiben (von denen nur wenige sich erhalten haben oder durch die beiden grundlegenden Briefeditionen H. Erlers und F. G. Jansens im Neudruck vorliegen). Das "Briefbuch" ist ein kostbares Authograph des Schumannhauses in Zwickau und noch unveröffentlich. Die Kombination beider Quellen, verbunden mit Eintragungen in die "Tagebücher" (von denen der erste Teil in einer dankenswerten Edition durch G. Eismann seit 1971 vorliegt), ermöglichte die Aufklärung vielfältig verflochtener geistiger Beziehungen, die in mancher Hinsicht ein neues Schumannbild erkennen lassen.

Ein kursorischer U̇berlick sei geboten: Der Dichter H. Chr. Andersen schreibt 1844 erfreut, daß seine "Glücksblume" Schumann "angesprochen" habe, in einem Postskript bemerkt er: "Meyerbeer war heute bei mir, viel- 
leicht schreibe ich für ihn eine Oper nach meinem Märchen: Das kleine Meerweib". Bettina von Arnim bekundet ihre "warme Teilnahme" und bezieht sich auf eine Vermittlung von Schumanns "bestem Freund", den jungen Geiger J. Joachim. Ergiebig ist ein längeres Schreiben der mit Mozarts Sohn befreundeten Komponistin Julie Webenau, geb. Baroni-Cavalcabò 1838. In schwerer Prüfungszeit empfing Schumann ermutigende Worte seines Freiberger Freundes Ernst Adolph Becker (1837). W. Sterndale Bennett schreibt 1837 begeistert über die "Symphonischen Etüden " op. 13. Neben Ludwig Berger ist $\mathrm{H}$. Berlioz mit einem noch unbekannten Schreiben vom 28. 12. 1836 von Interesse, in dem er sich für die Fürsprache in Schumanns Zeitschrift bedankt und von seinem kompositorischen Plänen einiges andeutet. Louis Böhner, ein skurriler Virtuose, der das Urbild des "Kapellmeisters Kreisler" E. T. A. Hoffmanns und Schumanns gewesen sein soll, fehlt mit einem langen Schreiben nicht (1834). Chelard, in seiner Zeit als Opernkapellmeister hochangesehen, ist auch mit Briefen an Clara vertreten. Lehrreich auch die Schreiben von dem großen Leipziger Geiger F. David und seiner Gattin, mit denen Schumanns sich herzlich begegneten: H. Dorn, Schumanns Lehrer, schreibt 1836: "Gestern erhielt ich Ihre neuesten Paganinis. Sie werden, lieber Schumann, eine gute Weile auf Verständnis und Anerkennung warten müssen. Wenn schon Chopin sich so langsam Bahn bricht, wie erst sein potenzierter Mitstrebender! Per aspera ad astra. Aber wozu Ihnen noch Mut einsprechen wollen? Wer Mut hat, so etwas zu schreiben, der besitat ihn auch, um in gleicher Weise fortzufahren. Mit heralicher Teilnahme, der Ihrige». Sehr eingehend hat sich dann Dorn erneut 1939 über den "Carnaval" op. 9 geäußert. F. J. Fétis, der belgische Musikforscher, erkundigt sich 1842 nach den Beständen der Leipziger Thomaskirchen-Bibliothek und erbittet Daten für einen Schumann-Artikel seiner Biographie universelle. Ernestine von Fricken, die Jugendfreundin, schreibt einen erschütternden Brief Ende Dezember 1837 als Antwort auf Schumanns Verlangen, alle seine Schreiben zurückzugeben; in einem kleinen Postskript lesen wir: "denkst du noch an die Etuden über daf Thema meines Pflegevaters?" (dies betrifft opus 13). Franz Glöggl erzählt aus Linz am 19. Juli 1838 von seinen Begegnungen mit Beethoven. ${ }^{6}$ Ottilie und Walther von Goethe (der letztere erhielt die "Davisdbündlertänze" dediziert) berichten aus dem Familienkreis. Der bedeutende Jenaer Philosoph F. Hand ist mit zwei Schreiben $(1835,1841)$ vertreten. Ein großartiger Huldigungsbrief von "3 Künstlern und einem Kunstliebhaber" ist am 26. 11. 1839 abgefaßt: Stephan Heller, Panofka, Albert Franck und Karl Halle richten eine enthusiastische Grußadresse an den Komponisten der "Kinderszenen" und des "Carnaval", nachdem sie diese Werke gerade gehört. Aus der frühen Zeit stammt ein Antwortbrief von J. N. Hummel, in dem kritisch zu dem wzuweilen schnell aufeinander folgenden Harmoniewechsel" und eine "bizzarre Originalität" Be-

\footnotetext{
' Hierzu vgl. bereits Verfasser, Neue Materialien zu $R$. Schumanns Wiener Bekanntenkreis, in: Stud. z. Mwiss. XXV, Wien 1962, S. 46 ff.
} 
zug genommen wird, die der "Schönheit, Klarheit» abträglich seien. Resigniert bemerkt der große österreichische Musikforscher v. Kiesewetter an Schumann als Redakteur seiner Zeeitschrift: "Gewiß, meine Herren, haben Sie nichts verloren, wenn Sie mich aufgeben«. Der Maler J. J. Bonaventure Laurens aus Montpellier (er zeichnete den Meister kurz vor dem geistigen Zusammenbruch) äußert sich zur "Sehnsucht" der romantischen Musik (April 1848). Zwei lange Briefe F. Liszts $(1838,1839)$ tragen zum »Carnaval" und den »Fantasiestücken" bei. Ein Schreiben des Jugendfreundes Willibald von der Lühe vom 27. 7. 1828 enthält den Urtext jener "ästhetischen Aphorismen", "Die Tonwelt" genannt, die wir als Autograph Schumanns kennen und die bereits eingehend für die Musikanschauung des jungen Romantikers gewürdigt worden sind: ${ }^{7}$ tatsächlich ist nun die Authentizität eingeschränkt und die literarische Provenienz geklärt. Von musiktheoretischem Interesse sind die Ausführungen von A. B. Marx über seine im Entstehen begriffene "Kompositionslehre" (1840). Ein Mendelssohn-Brief vom 12. 9. 1839 tritt hinzu, verbunden mit einem Schreiben der Mutter Lea Mendelssohn Bartholdy: "Mein Sohn schreibt mir so viel zu Ihrem Lobe, daß ich a'oppelt bedaure, Sie gestern verfehlt zu haben. Bitte, lassen Sie mich wissen, welchen Abend es Ihnen conveniert.... Über die fis-moll-Sonate opus 11 schreibt Ignaz Moscheles nach eingehendem Studium ausführlich mit zahlreichen Notenbeispielen, auch zur Spieltechnik; angeheftet ist jene, in der Schumannschen Zeitschrift veröffentlichte Besprechung des Werks, die zugleich eines der wichtigsten Dokumente der romanischen Beethovenästhetik darstellt. ${ }^{\text {Wilhelmine Schroeder-Devrient }}$ bekundet 1844: "Auch ist es mein großer Wunsch, die Lieder, die sich vorzugsweise für mich eignen, bevor ich sie öffentlich vortrage, Sie Ihnen vorgesungen zu haben, damit ich es Ihnen auch gewiß zu Danke mache«. I. X. v. Seyfried, einer der ersten, die sich für Schumann einsetzten, schließt ein längeres Schreiben 1840: "Gott sei gelobt! Beethoven ist für uns nich gestorben! Der Himmel verleihe dem Schöpfer des Paulus unvergängliche Kraft im Vollbringen". F. Silcher berichtet, es habe ihn «schon lange die Idee beschäftigt, Vergleichungen zwischen Beethovenscher Musik una Ossian'scher Poesie anzustellen." L. Spohr beglückwünscht den Komponisten der Frühlingssinfonie, stößt sich aber an den »krassen Begleitungsfiguren im Adagio», die »den Gesang zu sehr einwickeln«. S. Sulzer schreibt sehr schöne Worte der Anerkennung zu "Widmung", "Lotosblume॥, wich fühle mich gedrängt, meine Ideen über diese herrlichen Schöpfungen in den hiesigen Blättern öffentlich auszusprechen«. Die Freundin Henriette Voigt, Schumanns $» A s$-Dur-Seele", kündet den Tod des genialen Pianisten Ludwig Schunke (der Schumanns Tokkata empfing) 1834 an; ihr letzter Brief 1839 schwärmt "mit zitternder Hand" von der "wunderschönen Sonate« Schumanns. Zwei Tagebücher dieser wundersamen Frau

7 A. Schmitz, Anfänge der Ästhetik R. Schumanns, in: Zeitschr. f. Musikwiss. II, Leipzig 1919/1920, S. 535 ff. und III, 1920/1921, S. $111 \mathrm{ff}$.

A. Schering, Beethoven und die Dichtung, Berlin 1936, S. 57 ff. 
hat der Verfasser jüngst aus dem Besitz der Nachkommen ans Licht ziehen können: es sind "Schreib-Kalender" für 1835 und 1836, in denen Mendelssohn sehr oft erwähnt ist, eine Publikation dieser Textorte hat der Verfasser in Vorbereitung. Dort findet sich auch: "Abends Schumann uns besucht, der auf dem Flügel fantasierte", "Schumann spielte noch im Dunkeln", "Abends kam Schumann und spielte herrlich, war höchst poetisch und liebenswürdig. Viel gesprochen über Musik", "Schumann lange da. Schön gespielt« (1836). ${ }^{9}$

Neben diesem neuen Briefbestand von ca. 150 Einsendern sind zahlreiche musikalische Albumblätter, Zeichnungen (auch von der Hand Mendelssohns), Haarbüschel (u.a. von C. M. v. Weber, vom jungen Brahms), Blumensträuße in einem Herbarium überliefert. Als Kuriosum erscheint ein Albumblatt Paganinis für das Wunderkind Clara Wieck 1829 mit einem 5 stimmigen "Preludio" für Violino solo. Nur andeutend liefert der Verfasser hier einen Vorbericht einer Publikation- die zunächst nur die an Schumann gerichteten Briefe, also nur etwa die Hälfte aller Objekte betrifft.

Ein weiterer gewichtiger Fund ist ein kammermusikalisches Werk der Frühzeit. Schumann schreibt in seinem späten Tagebuch VIII (1846-1850): "Sehr gut erinnere ich mich einer Stelle in einer meiner Kompositionen, von der ich mir sagte, sie sei romantisch, wo ein von der alten Musik abweichender Geist sich mir eröffnete, ein neues poetisches Leben sich mir zu erschließen schien (es war das Trio eines Scherzo eines Klavierquartettes)". Hierbei hat Schumann das Wort wromantisch" unterstrichen. Den fraglichen Alternativsatz hat der Verfasser mit seiner schwebenden Harmonik als Notenbeispiel bekannt gemacht.10 Das ganze Werk aber, vier Sätze umfassend und im Frühjahr 1829 in Heidelberg entstanden, blieb bis heute unveröffentlicht. Das Autograph befindet sich seit mehreren Jahrzehnten in Privatbesitz (Sammlung Wiede in Zwickau-Weißenborn bis 1947, sodann an verschiedenen Orten in Niederbayern), war praktisch unzugänglich und ist 1974 von der Universitätsbibliothek Bonn erworben worden. Der Verfasser übernahm im Auftrag der Erben die Expertise und hatte seit 1937, seiner ersten Identifikation des Werkes, die Möglichkeit, den Notentext zu prüfen und mit anderen Fragmentquellen zu ergänzen. Mit einem detaillierten Kritischen Bericht und einer biographischen Dokumentation erscheint es 1978 im Verlag Heinrichshofen (Wilhelmshaven) als Beiheft der "Quellenkataloge zur Musikgeschichte». Nur schemenhaft, mit

\footnotetext{
' Weitere Hinweise jüngst in Verfasser, $R$. Schumanns Klavierwerke, Neue biographische und textkritische Untersuchungen, Band I, Opus 1-6, Wilhelmshaven 1976, S. 163. Ferner vgl. den Überblick des Verfassers in: D. Shitomirski, R. A. Schumann, Briefe, S. 663-669, Quellen zur Briefedition (russisch), Moskau 1970 (Muzika).

${ }^{10}$ Verfasser, Einfïhrung..., 1939 (1941), S. 354. Neuerdings hat K. H. Wörner ( $R$. Schumann, Zuirich 1949, S. 39 f.) dieses Notenbeispiel wiederabgedruckt und in verdienstlicher Weise auf dieses "romatische» Zitat verwiesen.
} 
abweichender Tonartangabe ist bisher im Schrifttum vom Werk die Rede.11 In dem frühen Tagebuch erscheint bereits am 1. Dezember 1828 die Eintragung: "Mein Quariett - Schubert ist tot - Bestürzung". Tatsächlich ist das Quartett unter starkem Einfluß Schuberts entstanden. "Mein Quartett« heißt es mehrmals am 1., 2., 3., 5. Februar 1929, am 4. Februar: "glückliche Fantasie», am 6. Februar bereits: "Ausschreibung der Stimmen für mein Quartett." Am Folgetage nennt er das Werk "Opus V", "Lobeserhebunden " habe ihm das Werk im Freundeskreis eingebracht. In einer häuslichen "Quartettvereinigung" (der 11, der Reihe) wurde es neben Kammermusik von C. M. v. Weber und Onslow gegeben. Das "Trio" vergleicht er mit einem "Sonnenaufgang". Die befreundete Gattin des berühmten romantischen Arztes E. A. Carus nahm herzlich am Enstehen des Klavierquartetts teil. Unmittelbar vor Beginn der Entwürfe zu opus 1, den "Abegg-Variationen ", war das Werk abgeschlossen; die über den Namen der Freundin Meta Abegg komponierten virtuosen Variationen wurden den "Alexandermarschvariationen" des I. Moscheles nachgebildet, die er ab 17. März 1829 studierte. Die Komposition Moscheles', ein Konzertstück für Klavier und Orchester, weist eine lange Orchesterintroduzione auf, die Modell für Schumanns Abegg-Variationen gestanden hat: diese waren ursprünglich auch als Konzertstück disponiert und die getilgte Introduzione mit verminderten Septakkorden und schwellenden Vorhaltklängen hat der Verfasser aus den Skizzenbüchern jüngst in Erfahrung gebracht.12 Über den Finalsatz schreibt Schumann im Tagebuch: "Fröhliche Wildheit darinnen, die in einer ganz anderen Welt noch einmal freundlich an die Vergangenheit denkt..., lobendes ästhetisches Urteil über das Ganze - das ganze Quartett hintereinander». Dieses Frühwerk, dessen Uraufführung im Frühjahr 1978 im Schumann-Gedenkzimmer in Bonn-Endenich erfolgte, ist der wichtigste Anreger zum genialen Klavierschaffen des jungen Schumann überhaupt. Zahlreich sind die Anklänge, namentlich in den "Papillons" (Finale mit dem punktierten Rhythmus), den "Intermezzi« (Sechzehntelgruppen in tiefer Lage). Eine Kette von unaufgelösten Septakkorden bei U̇berleitungsstellen im Finale und Sekundreibungen nehmen die berühmte "Mondnacht" vorweg. Einiges hat bereits Hans F. Redlich, dem der Verfasser Teile des unbekannten Werks zum Studium überließ, in verdienstvoller Weise als Parallelfall nachgewiesen. ${ }^{13}$

" In der verdienstlichen Teiledition der Tagebücher durch G. Eismann, Band I, Leipzig 1971, ist im Register das Werk nur mit Fragezeichen geführt, Eismann zitiert nach dem "Projektenbuch" falsch »e-moll» (R. Schumann..., Leipzig 1956, S. 81, analog im älteren und jüngeren Schrifttum, vgl. W. V. Wasielewski, $R$. Schumann, 4. Aufl., Leipzig 1904, S. 45, P. und W. Rehberg, R. Schumann, Zürich-Stuttgart 1954, S. 727 etc.).

${ }^{12}$ Vollständig wiedergegeben nach dem autographen Klavierauszug in Verfasser, $R$. Schumanns Klavierwerke..., S. 37-39.

${ }^{13}$ Hans F. Redlich, Schumann discoveries, in: Monthly Musical Record LXXX/LXXXI, London 1950/1951, S. 143 ff., 182 ff., 261 ff., ferner A Postscript, ibid., LXXXI, S. 14 ff. 
Natürlich steht mit dem c-moll-Thema des ersten Satzes Beethovens letzte Klaviersonate opus 111 Pate, der Satz ist aber auch von op. 81a ("Les Adieux") angeregt, ferner von der zuckenden Motivsprache der IX. Sinfonie und deren Monumentalthematik. Neben den "Sechs frühen Liedern" (Wien 1935) und den "Acht Polonaisen für Pianoforte zu 4 Händen« (Wien 1933), deren Edition wir Karl Geiringer verdanken, ferner den nur fragmentarisch überlieferten "Variationen über das Allegretto-Thema aus Beethovens VII. Sinfonie» (Duisburg-München 1977, ed. Karl Müntzer) ist das neue Quartett, was Umfang und Wert betrifft, wohl der bedeutendste Fund, der uns die stilistische Eingangsphase des großen Klavierwerks des Jünglings näher bestimmen läßt. Merkwürdig ist die Konzeption eines ensemble-Klangs, aus dem sich die spezifische Klavierproduktion dann entfalten sollte; nicht unerheblich wirkten auch frühe Lieder mit, wie der Einzelfall einer "Aria" (langsamer Satz der fis-moll-Sonate opus 11) deutlich macht. Die pianistische Evolution zehrte bis 1836 von dem nun ermittelten Qaurtettsatz, was ergänzend die zahlreichen Skizzenfragmente in den Entwurfsheften (ehemals Sammlung Wieder, jetzt ebenfalls Universitätsbibliothek Bonn) erkennen lassen, deren systematische Auswertung der Verfasser in Kürze vorlegen wird. ${ }^{14}$ Nicht zuletzt ist damit eine weitere Kontrollinstanz für den Aufbau eines "Urtextes" der Klavierwerke des Meisters geboten. Denn die alte "Gesamt-Ausgabe» unter der Edition Clara Schumanns, vor ca. 100 Jahren vorgelegt, orientierte sich nicht am Skizzenapparat, ja zog nicht einmal in jedem Fall den Erstdruck zu Rate, sondern folgte apokryphen Quellen, was eine Nachprüfung zeigt. Der Verfasser ist gegenwärtig damit beschäftigt, aufgrund der Handexemplare Schumanns, de z. T. autographe Korrekturen enthalten (Erstdrucke), in Verbindung mit dem verstreuten handschriftlichen Apparat (z. T. auch Kopistenfassungen) einen Urtext zu restituieren, wobei in mehreren Fällen auch die Früh- und Spätfassung getrennt vorzulegen ist. Damit wird das Resultat einer wissenschaftlichen Quellenkritik auch dem praktischen Spieler erschlossen..$^{15}$

\section{POVZETEK}

V prispevku je prvič opisan literarni in muzikalni vir za poznavanje ustvarjanja Roberta Schumanna. Gre za "Družinski album», ki poleg risb - nekatere so Mendelssohnove - spominskih predmetov (tudi lasje C. M. Webra, mladega Brahmsa), spominskih listov, glasbenih rokopisov, vsebuje okoli 150 na Schumanna naslovljenih pisem, ki so do leta 1945 bila $\mathrm{v}$ Berlinu, a so poslej izginila. To literarno gradivo nudi marsikateri nov vpogled $\mathrm{v}$ življenje in delo skladatelja, prav tako pa osvetljuje prijateljske glasbenike, pesnike in slikarje, zlasti tiste, S katerimi je bila 1840 povezana skladateljeva žena Clara. - Najpomembnejša

${ }^{14}$ Band II des Anm. 9 und 12 genannten Werkes, betreffend Opus 7-17, Wilhelmshaven 1979.

${ }_{15} R$. Schumann, Klavierwerke, Urtextausgabe, Neue Revision, ed. vom Verfasser, Duisburg-München (Henle-Verlag). Erschienen sind seit 1975: op. 1, 2, 4, $6,7,9,11,12,13,15,16,17,18,19,21,23,26,28,68,99,111,124$. In Vorbereittung op. 22, 82. 
najdba na glasbenem področju je klavirski kvartet v c-molu, v štirih stavkih, ki je nastal spomladi 1829 in ki ga je Schumann sistematično izkoristil $v$ svojih genialnih klavirskih delih prvega obdobja, namreč $v$ op. 1, 2, 4, 9, 11 (zavoljo česar je opustil misel na objavo). To komorno delo označuje Schumanna v svojem poznem dnevniku 1846-50 kot prvo "romantično» predslutnjo "nove" glasbe, tako da imamo tukaj ne glede na povečan glasbeni repertoar opraviti s posebnim dokumentom $v$ zvezi $\mathrm{z}$ nastankom romantičnega stila. Končno osvetljuje tudi probleme $\mathrm{v}$ zvezi $\mathrm{z}$ izdajo klavirskih del na podlagi prvotnih rokopisov nasproti "celotni izdaji», ki jo je pred kakimi 100 leti brez komentarja opravila Clara Schumann. Osnovo tvori sedaj zbirka prvotiskov, ki so se ohranili kot skladateljevi avtorski izvodi, in ogromen fond rokopisov, med katerimi izstopa 5 skicirnih zvezkov, ki so pred kratkim iz privatnih rok prišli v univerzitetno knjižnico $\mathrm{v}$ Bonnu. Te raziskave, o katerih izide poročilo, so $\mathrm{v}$ teku. 